\title{
Mismanagement of obstructive sleep apnea may result in finding these patients dead in bed
}

\author{
Jonathan L. Benumof, MD
}

Received: 6 July 2015 / Revised: 3 August 2015 / Accepted: 8 October 2015 / Published online: 19 October 2015

(C) Canadian Anesthesiologists' Society 2015

The first step in fixing a problem is identifying the problem. Accordingly, this issue of the Journal includes a survey by Cordovani et al. on the perioperative management of patients with obstructive sleep apnea (OSA) which proves very helpful in that regard. ${ }^{1}$ The authors found that approximately half of Canadian anesthesiologists screen for OSA, and approximately half work in a hospital with a functional OSA policy. In my opinion, these main findings most probably correspond with those in the USA. On the one hand, these findings represent an improvement when compared with just 15-20 years ago - there has been a large increase in the number of doctors who systematically screen for OSA and an increase in the number of institutions that have a functional OSA management policy-and such practices decrease the chance of OSA mismanagement. On the other hand, many anesthesiologists still do not screen for OSA and many institutions do not have a working OSA policy, and such practices increase the chance of OSA mismanagement. The real problem with the lack of OSA screening and institutional OSA management policy is that these institutions are largely ignoring the OSA disease, and as a result, they may find some postoperative OSA patients dead in bed (DIB).

As a medical expert witness over the past 15 years (from 1999-2014), I have had 12 closed medical malpractice cases wherein postoperative OSA patients have been found DIB within $48 \mathrm{hr}$ of surgery. Each one of these 12 cases clearly shows the lethal consequence of not knowing that a patient has OSA (i.e., no systematic identification) and/or

J. L. Benumof, MD ( $\varangle)$

Department of Anesthesiology, UCSD Medical Center, 402

Dickinson Street, Mail Code 0801, San Diego, CA 92103, USA

e-mail: jbenumof@ucsd.edu ignoring the clinical significance of a patient having OSA (i.e., no functional institutional policy). The findings in these 12 cases are entirely consistent with the findings of a recent review of the legal literature regarding complications in 24 OSA patients undergoing surgery. ${ }^{2}$ The Table (in aggregate form to preserve patient anonymity) details my 12 cases.

From the Table, the prototypical OSA-DIB case is as follows: A 58-yr-old continuous positive airway pressure (CPAP)-compliant male $(170 \mathrm{~cm}, 120 \mathrm{~kg}$, body mass index $40 \mathrm{~kg} \cdot \mathrm{m}^{-2}$ ) with polysomnography (PSG)-proven severe OSA undergoes orthopedic, upper airway, or abdominal surgery under general anesthesia. The patient has an uncomplicated stay in the postanesthesia care unit until discharged to an unmonitored bed without CPAP or oxygen. After receiving small (and within standard of care) doses of narcotics for pain for $11 \mathrm{hr}$, the patient is found DIB. Advanced cardiac life support is either not attempted or fails to return the patient to their baseline state of life.

How frequently are postoperative OSA patients found DIB? I do not know - and to my knowledge, neither does anyone else - but a great effort is underway to find out. The Society of Anesthesia and Sleep Medicine (SASM) and the American Society of Anesthesiology (ASA) Closed Claims Project have together established an OSA Death and Near Miss Registry ${ }^{\mathrm{A}}$ in the hope of collecting a large number of detailed case reports for analysis. In a few years, it may be possible to get some idea of the numerator (i.e., DIB and near miss cases) and the denominator (i.e., postoperative OSA patients managed in specific ways).

\footnotetext{
$\bar{A}$ Closed Claims Project and its Registries. Available from URL: http://www.asaclosedclaims.org (accessed August 2015).
} 
Table Aggregate details of my 12 closed medicolegal cases of finding obstructive sleep apnea patients dead in bed

\begin{tabular}{|c|c|}
\hline Data Heading & Data \\
\hline Age, Sex, Body Habitus & $\begin{array}{l}\text { The ages ranged from } 27-74 \mathrm{yr} \text {, with a distribution skewed towards the } 50-60 \\
\text { years interval. Male } / \mathrm{Female}=8 / 4 \text { and height, weight, BMI ranges were } 158- \\
180 \mathrm{~cm}, 61-149 \mathrm{~kg} \text {, and } 24-54 \mathrm{~kg} \cdot \mathrm{m}^{-2} \text {, respectively. One outlier had a weight } \\
=61 \mathrm{~kg} \text { and } \mathrm{BMI}=24 \mathrm{~kg} \cdot \mathrm{m}^{-2} \text { in an otherwise normal distribution of values. }\end{array}$ \\
\hline Basis for OSA diagnosis & $\begin{array}{l}10 \text { patients had a positive PSG study; } 2 \text { patients had only a strong history } \\
\text { consistent with OSA }\end{array}$ \\
\hline OSA Severity & $\begin{array}{l}9 \text { patients had severe OSA by PSG; } 1 \text { had moderate OSA by PSG; } 2 \text { had } \\
\text { moderate to severe OSA by history }\end{array}$ \\
\hline Likely Cause of OSA & OSA was related to obesity in 11 cases and retrognathia in 1 case \\
\hline Preoperative CPAP use & 7 were on CPAP preoperatively \\
\hline Surgery Performed & $\begin{array}{l}3 \text { were orthopedic, } 6 \text { were abdominal ( } 3 \text { open, } 3 \text { laparoscopic), and } 3 \text { were upper } \\
\text { airway }\end{array}$ \\
\hline Anesthesia Administered & All 12 had general anesthesia. 1 required a fibreoptic intubation. \\
\hline PACU Problems & $\begin{array}{l}8 \text { patients had no problems in the PACU. } 1 \text { patient had a few observed apneas } \\
\text { considered to be WNL. } 1 \text { patient had } 2 \text { apneas }\left(\mathrm{SpO}_{2}=82 \%\right) \text { with } \\
\text { spontaneous recovery to WNL. } 1 \text { patient received small doses of narcotics } \\
\text { over } 1 \text { hour, had a witnessed arrest in the PACU, short ACLS time, but } \\
\text { suffered brain damage. } 1 \text { patient received } 2.5 \mathrm{mg} \text { hydromorphone over } 90 \\
\text { min and was found cyanotic and unarousable in the PACU, had ACLS but } \\
\text { suffered brain damage. }\end{array}$ \\
\hline
\end{tabular}

Disposition of the 10 patients discharged from the PACU in All 10 patients went to an unmonitored bed; 7 went to a ward and 3 went home. WNL condition

$\mathrm{O}_{2}$ and CPAP for the 10 patients who were discharged from the None were on $\mathrm{O}_{2}$; none were on CPAP.

PACU in WNL condition

Time from PACU Discharge to being found DIB

Narcotics administered (after PACU discharge) to the 10 patients discharged from the PACU in WNL condition

ACLS Outcome after being found DIB

Autopsy findings

\section{2-29 hr, with an even distribution of time values throughout this range \\ 1 patient did not receive narcotics; 3 patients received very small doses; 4 patients received small doses; and 2 patients were on standard PCA with basal rates of $0.5 \mathrm{mg} \cdot \mathrm{hr}^{-1}$ and $1.0 \mathrm{mg} \cdot \mathrm{hr}^{-1}$}

In 3 cases, ACLS was not even attempted, and in 4 cases, ACLS failed. In 5 cases, ACLS was initially successful, but all 5 patients had hypoxic encephalopathy, and 4 of the 5 patients died within 21 days. The 1 patient who survived with brain injury beyond 21 days had pulmonary edema on chest $x$-ray immediately after receiving a brief period of ACLS.

An autopsy was performed in 4 cases; in 3 of the cases, the autopsy findings were consistent with NPPE.

ACLS = Advanced Cardiac Life Support BMI = body mass index; CPAP = continuous positive airway pressure; NPPE = negative pressure pulmonary edema; PACU = postanesthesia care unit; OSA = obstructive sleep apnea; $\mathrm{PCA}=$ patient-controlled analgesia; PSG = polysomnography; $\mathrm{WNL}=$ within normal limits

What are the lessons to be learned from the Cordovani et al. survey, ${ }^{1}$ the above 12 tragic cases, and the prototypical OSA-DIB case (derived from the 12 cases)? First and foremost, if surgical patients with PSG-proven severe OSA (nine of the above 12 cases), moderate OSA (one of the above 12 cases), or only a strong history suggestive of OSA (two of the above 12 cases) are sent to an unmonitored environment postoperatively (i.e., all 12 of the above cases), some will most certainly be found DIB. Institutions could abort going down the pathway of unmonitored beds if caregivers screened for OSA and had institutional policies that put these patients in monitored beds. The take-home message of this editorial is that all practitioners in all disciplines should understand that patients with significant OSA having significant surgery should go to a monitored bed as recommended by the ASA OSA Guidelines on the

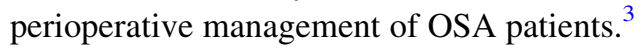

Second, all 12 cases were off CPAP and off oxygen at the time they were found DIB. An OSA case off CPAP and off oxygen should be a strong relative indication for the patient to go to a monitored environment.

Third, in seven of the 12 cases, there were either failed attempts or no attempts at ACLS resuscitation, indicating that the patient had been DIB for a long time.

Fourth, in the context of postoperative patients with significant OSA, significant obesity, and off CPAP and oxygen administering only a relatively low dose of narcotics does NOT preclude finding patients DIB.

Fifth, the finding of pulmonary edema in four of the 12 cases deserves special comment. The autopsy evidence for 
pulmonary edema in three cases consisted of (a) very heavy lung weights, (b) lung surfaces that exuded edema fluid, (c) tracheobronchial trees that contained pink frothy fluid, (d) interstitial and alveolar edema on microscopy, and in one case, $x$-ray findings of pulmonary edema. Since the mechanism of death was, in all probability, obstruction of the upper airway, a terminal unsuccessful attempt to breathe against the obstruction could cause negative pressure pulmonary edema (NPPE). Most importantly, none of these four patients had any other possible reason to have pulmonary edema as shown by pre-death clinical data or post-death autopsy findings. I interpret the pulmonary edema accompanying the terminal crisis in these four cases as NPPE.

Finally, there were no OSA-DIB cases in patients with mild OSA; it makes sense that mild OSA entails less risk of being found DIB than severe OSA.

The Cordovani survey ${ }^{1}$ tells us that we have a potential OSA management problem. The 12 cases I presented in this editorial, the prototypical case derived from these 12 cases, and the legal literature review ${ }^{2}$ tell us that we do have an OSA mismanagement problem. It is simply common sense that, if a morbidly obese patient with severe OSA is given a painful incision and narcotics and is then placed in an isolated unmonitored environment off CPAP and off oxygen, there is a definite chance that the patient will stop breathing (or be unable to breathe against an obstruction) and be found DIB. The full extent of this likelihood awaits the results of a cohort analysis from the OSA Death and Near Miss Registry. Until further study offers better information, we need to systematically identify these patients and treat them with appropriate institutional policies.

\section{La mauvaise gestion du syndrome d'apnée-hypopnée du sommeil pourrait se traduire par la découverte de ces patients morts dans leur lit}

La première chose à faire pour régler un problème est d'identifier ce problème. C'est pourquoi vous trouverez dans ce numéro du Journal une enquête réalisée par Cordovani et coll. sur la gestion périopératoire des patients atteints de syndrome d'apnée-hypopnée du sommeil (SAHS) qui s'avère très utile de ce point de vue. ${ }^{1}$ Les auteurs ont constaté qu'environ la moitié des anesthésiologistes canadiens recherchent l'existence d'un
SAHS et qu'environ la moitié travaillent dans un hôpital doté d'une politique fonctionnelle en matière de SAHS. À mon avis, ces principales constatations correspondent probablement à la situation existant aux États-Unis. D'un côté, elles représentent une amélioration par rapport à ce qu'il se passait il y a juste 15 ou 20 ans (il y a eu une très nette augmentation du nombre de médecins recherchant systématiquement le SAHS et du nombre d'établissements disposant d'une politique fonctionnelle de gestion du SAHS) et ces pratiques diminuent le risque de mauvaise gestion du SAHS. D'un autre côté, de nombreux anesthésiologistes ne dépistent toujours pas le SAHS et de nombreux établissements n'ont toujours pas de politique active sur le SAHS et ces pratiques augmentent le risque de mauvaise gestion du SAHS. Le vrai problème avec le manque de dépistage du SAHS et de politique de gestion du SAHS au niveau de l'établissement est que les établissements ignorent quasi complètement ce syndrome et que, par conséquent, ils peuvent parfois retrouver en postopératoire certains patients atteints de SAHS morts dans leur lit (MDL).

Ayant témoigné en tant qu'expert médical au cours des 15 dernières années (de 1999 à 2014), j'ai eu à connaître 12 cas de fautes médicales où des patients atteints de SAHS ont été retrouvés morts dans leur lit, dans les 48 h suivant une intervention chirurgicale. Chacun de ces 12 cas illustre clairement la mortelle conséquence de ne pas savoir qu'un patient est atteint de SAHS (c'est-à-dire, aucune identification systématique) et/ou d'ignorer ce que signifie cliniquement pour un patient l'existence du SAHS (c'est-à-dire, l'absence de politique fonctionnelle dans l'établissement). Dans ces 12 cas, les constatations concordent totalement avec les constations d'une analyse récente publiée dans le domaine du droit et concernant les complications survenues chez 24 patients atteints de SAHS subissant une intervention chirurgicale. ${ }^{2}$ Le Tableau présente sous forme agrégée (pour préserver l'anonymat des patients) les détails de mes 12 cas.

Le Tableau permet de dresser un portrait type du cas de SAHS MDL: homme âgé de 58 ans répondant à une pression positive continue des voies aériennes (CPAP), mesurant $170 \mathrm{~cm}$ pour $120 \mathrm{~kg}$ (indice de masse corporelle $40 \mathrm{~kg} \cdot \mathrm{m}^{-2}$ ) atteint de SAHS prouvé par polysomnographie (PSG) subissant une chirurgie orthopédique, des voies aériennes hautes ou abdominale sous anesthésie générale. Le patient n'a présenté aucune complication pendant son séjour en salle de réveil jusqu'à son transfert dans un lit non contrôlé, sans CPAP ou oxygène. Après avoir reçu de petites doses (dans la limite des normes de soins) de narcotiques contre la douleur pendant $11 \mathrm{~h}$, le patient est retrouvé mort dans son lit. La réanimation cardiaque avancée est soit non tentée ou ne parvient pas à ramener le patient à la vie. 
Tableau Données agrégées de mes 12 cas médicolégaux (clos) de patients atteints de syndrome d'apnée-hypopnée du sommeil, trouvés morts dans leur lit

\begin{tabular}{|c|c|}
\hline Type de données & Détail des données \\
\hline Âge, Sexe, corpulence & $\begin{array}{l}\text { Âges compris entre } 27 \text { et } 74 \text { ans avec une distribution principalement entre } 50 \text { et } 60 \text { ans. } \\
\text { Ratio hommes/femmes: } 8 / 4 \text {; taille, poids, IMC: compris respectivement entre } 158 \\
\text { et } 180 \mathrm{~cm}, 61 \text { et } 149 \mathrm{~kg} \text {, et } 24 \text { et } 54 \mathrm{~kg} \cdot \mathrm{m}^{-2} \text {. Un cas extrême avait un poids de } 61 \mathrm{~kg} \text { et } \\
\text { un IMC de } 24 \mathrm{~kg} \cdot \mathrm{m}^{-2} \text {; la répartition des autres valeurs étant par ailleurs normales. }\end{array}$ \\
\hline Base du diagnostic de SAHS & $\begin{array}{l}\text { Chez } 10 \text { patients, la PSG a été positive; chez } 2 \text { patients, seule l'anamnèse était fortement } \\
\text { compatible avec un diagnostic de SAHS }\end{array}$ \\
\hline Sévérité du SAHS & $\begin{array}{l}9 \text { patients avaient un SAHS sévère déterminé par la PSG; } 1 \text { patient avait un SAHS modéré } \\
\text { déterminé par la PSG; } 2 \text { patients avaient un SAHS modéré d'après l'anamnèse. }\end{array}$ \\
\hline Cause probable du SAHS & Le SAHS était lié à l'obésité dans 11 cas et à une rétrognathie dans 1 cas. \\
\hline Utilisation préopératoire de la CPAP & 7 patients étaient sous CPAP en préopératoire. \\
\hline Nature de l'intervention chirurgicale & $\begin{array}{l}3 \text { chirurgies orthopédiques, } 6 \text { chirurgies abdominales ( } 3 \text { ouvertes, } 3 \text { laparoscopiques), et } \\
3 \text { chirurgies des voies aériennes hautes. }\end{array}$ \\
\hline Anesthésie administrée & $\begin{array}{l}\text { Les } 12 \text { patients ont eu une anesthésie générale. } 1 \text { patient a nécessité une intubation sous } \\
\text { bronchoscopie. }\end{array}$ \\
\hline Problèmes en salle de réveil & $\begin{array}{l}8 \text { patients n'ont eu aucun problème en salle de réveil. Chez } 1 \text { patient, quelques rares } \\
\text { apnées ont été observées et jugées dans les limites de la normale. } 1 \text { patient a fait } \\
2 \text { apnées }\left(\mathrm{Sp}_{2}=82 \%\right) \text { avec récupération spontanée dans les limites de la normale. } \\
1 \text { patient a reçu de petites doses de narcotiques pendant } 1 \text { heure et un arrêt cardiaque } \\
\text { observé dans la salle de réveil; la durée de la ressuscitation cardiaque avancée a été } \\
\text { brève, mais le patient a présenté une lésion cérébrale. } 1 \text { Patient a reçu } 2,5 \mathrm{mg} \\
\text { d'hydromorphone sur une période de } 90 \text { min et a été trouvé cyanosé, impossible à } \\
\text { réveiller en salle de réveil, a bénéficié d'un ACLS mais a présenté une lésion cérébrale. }\end{array}$ \\
\hline $\begin{array}{l}\text { Suite des soins pour les } 10 \text { patients ayant quitté la } \\
\text { salle de réveil dans un état dans les limites de la } \\
\text { normale }\end{array}$ & $\begin{array}{l}\text { Les } 10 \text { patients ont été mis dans un lit non monitoré; } 7 \text { sont restés hospitalisés et } 3 \text { sont } \\
\text { rentrés chez eux. }\end{array}$ \\
\hline
\end{tabular}

$\mathrm{O}_{2}$ et CPAP pour les 10 patients ayant quitté la salle Aucun n'a reçu d' $\mathrm{O}_{2}$; aucun n'a eu de CPAP.

de réveil dans un état dans les limites de la normale

Délai écoulé entre le congé de la salle de réveil et la 2 à 29 h, avec une répartition égale dans cette plage horaire. constatation du décès

Narcotiques administrés (après le congé de la salle de réveil) aux 10 patients ayant quitté la salle de réveil dans un état dans les limites de la normale

Résultat de la tentative de ressuscitation après avoir trouvé le patient mort dans son lit

Constatations à l'autopsie
1 patient n'a pas reçu de narcotique; 3 patients ont reçu de très petites doses; 4 patients ont reçu de petites doses; et 2 patients ont reçu une PCA usuelle avec des taux de base de $0,5 \mathrm{mg} \cdot \mathrm{h}^{-1}$ et $1,0 \mathrm{mg} \cdot \mathrm{h}^{-1}$

La ressuscitation avec ACLS n'a même pas été tentée dans 3 cas et elle a échoué dans 4 cas. Elle a initialement réussi dans 5 cas, mais ces 5 patients présentaient une encéphalopathie hypoxique et 4 d'entre eux sont décédés dans les 21 jours suivants. Le patient ayant survécu au-delà de 21 jours avec une lésion cérébrale avait un œdème pulmonaire visible sur la radiographie de thorax immédiatement après avoir bénéficié d'un ACLS de courte durée.

Une autopsie a été pratiquée dans 4 cas; dans 3 cas, les constatations de l'autopsie étaient compatibles avec un NPPE.

ACLS: système évolué de maintien des fonctions cardiaques; IMC: indice de masse corporelle; CPAP: pression positive continue des voies respiratoires; NPPE: œdème pulmonaire à pression négative; SAHS: syndrome d'apnée-hypopnée du sommeil; PCA: analgésie contrôlée par le patient; PSG: polysomnographie

À quelle fréquence retrouve-t-on en postopératoire des patients atteints de SAHS morts dans leur lit? Je l'ignore et, à ma connaissance, personne ne le sait, mais de gros efforts sont actuellement déployés pour le découvrir. La Society of Anesthesia and Sleep Medicine et le projet Closed Claims Project de l'American Society of Anesthesiology (ASA) ont créé ensemble un registre OSA Death and Near Miss Registry ${ }^{\mathrm{A}}$ (registre des décès et décès évités de justesse en rapport avec un SAHS) dans l'espoir de collecter un grand nombre de rapports de cas détaillés à analyser. Dans quelques années, il sera peut-être possible d'avoir une idée du numérateur (c'est-à-dire, le nombre de cas de MDL et de décès évités de justesse) et le dénominateur (c'est-à-dire, le nombre de patients atteints de SAHS en postopératoires et pris en charge de façon spécifique). 
Quels enseignements pouvons-nous tirer de l'enquête de Cordovani et coll., ${ }^{1}$ des 12 cas tragiques ci-dessus et du cas type de SAHS-MDL (tiré des 12 cas)? D'abord et avant tout, si des patients chirurgicaux ayant un SASH sévère prouvé par PSG (neuf des 12 cas ci-dessus), un SAHS modéré (un des 12 cas ci-dessus) ou seulement des antécédents suggérant fortement un SAHS (deux des cas ci-dessus) sont envoyés dans un environnement postopératoire non monitoré (ici, les 12 cas précités), certains seront très certainement retrouvés morts dans leur lit. Les établissements pourraient éviter de s'engager dans la voie de lits non monitorés si les professionnels de soins de santé dépistaient le SAHS et si des politiques d'établissement dirigeaient ces patients dans des lits monitorés. Le message à retenir de cet éditorial est que tous les praticiens, dans toutes les disciplines, doivent comprendre que des patients atteints de SAHS significatif subissant une intervention chirurgicale significative doivent être mis dans un lit monitoré, tel que le recommandent les lignes directrices de l'ASA sur le SAHS pour la gestion périopératoire des patients atteints de ce syndrome. ${ }^{3}$

Deuxièmement, au moment où ils ont été trouvés MDL, les 12 patients n'avaient ni oxygène, ni CPAP. Un cas de SAHS sans oxygène et sans CPAP constituerait une indication relativement forte de mise de ce patient dans un environnement surveillé.

Troisièmement, dans sept des 12 cas, il y a eu échec ou absence de tentative de ressuscitation cardiaque avancée, indiquant que le patient était décédé depuis déjà un certain temps.

Quatrièmement, dans le contexte de patients postopératoires ayant un SAHS significatif, une obésité significative, sans CPAP ni oxygène, l'administration de narcotiques à faibles doses seulement, n'empêche PAS de découvrir des patients morts dans leur lit.

Cinquièmement, la découverte d'un œdème pulmonaire dans quatre des 12 cas mérite une mention particulière. Dans trois cas, les constatations faites à l'autopsie incluaient (a) le poids très important des poumons, (b) l'exsudation de liquide d'œdème à la surface des poumons, (c) la présence d'un liquide rose mousseux dans l'arbre trachéobronchique, (d) un œdème alvéolaire et interstitiel à l'examen microscopique; et dans un cas l'examen radiologique a mis en évidence des signes d'œdème pulmonaire. Considérant que le mécanisme responsable du décès était, très probablement, une obstruction de la voie aérienne haute, l'échec d'une tentative terminale de respirer en dépit de l'obstruction provoquerait un œè̀me pulmonaire à pression négative (NPPE). Plus important, aucun de ces quatre patients n'avait d'autre raison possible de faire un œdème pulmonaire comme le montraient les données cliniques antérieures aux décès ou les constatations autopsiques. J'interprète donc l'œdème pulmonaire accompagnant la crise terminale chez ces quatre patients comme étant un NPPE.

Enfin, il n'y a pas eu de cas de MDL lié au SAHS chez des patients ayant des formes légères du syndrome; il est logique qu'un SAHS léger expose moins au risque d'être retrouvé mort dans son lit qu'un SAHS sévère.

L'enquête de Cordovani ${ }^{1}$ nous indique que nous avons un problème potentiel de gestion du SAHS. Les 12 cas que j'ai présentés dans cet éditorial, le cas type tiré de ces 12 cas et l'analyse des publications légales ${ }^{2}$ nous indiquent que nous avons un problème de mauvaise gestion du SAHS. C'est simplement faire preuve de bon sens de dire qu'un patient ayant une obésité morbide, un SAHS sévère, une incision douloureuse et des narcotiques, puis placé dans un environnement isolé non monitoré, sans CPAP ni oxygène, court un risque certain d'arrêt respiratoire (ou qu'il se trouve dans l'impossibilité de respirer en cas d'obstruction de la voie aérienne) et qu'il sera trouvé mort dans son lit. Toute l'ampleur de cette probabilité ne sera connue qu'avec les résultats de l'analyse de cohorte du registre des décès et décès évités de justesse en rapport avec un SAHS (OSA Death and Near Miss Registry). En attendant que d'autres études nous fournissent une meilleure information, nous devons identifier systématiquement et traiter ces patients en suivant des politiques d'établissement adaptées.

Conflicts of interest None declared.

Conflits d'intérêts Aucun déclaré.

\section{References}

1. Cordovani L, Chung F, Germain G, et al. Perioperative management of patients with obstructive sleep apnea: a survey of Canadian anesthesiologists. Can J Anesth 2016; 63: this issue: DOI: 10.1007/s12630-015-0512-y.

2. Fouladpour N, Jesudoss R, Bolden N, Shaman Z, Auckley D. Perioperative complications in obstructive sleep apnea patients undergoing surgery: a review of the legal literature. Anesth Analg 2015: DOI: 10.1213/ANE.0000000000000841.

3. Gross JB, Bachenberg KL, Benumof JL, et al. Practice guidelines for the perioperative management of patients with obstructive sleep apnea: a report by the American Society of Anesthesiologists Task Force on the Perioperative Management of Patients with Obstructive Sleep Apnea. Anesthesiology 2006; 104: 1081-93. 